\title{
Avaliação do Tratamento Clínico da Endometriose
}

\author{
Clinical Treatment Evaluation of Endometriosis
}

\author{
Marcos Dias de Moura, Taíse de Negreiros Pereira, \\ Antonio Alberto Nogueira, Rui Alberto Ferriani, \\ Maria Matheus de Sala, Rosana Maria dos Reis
}

\begin{abstract}
RESUMO
Objetivo: o objetivo do presente trabalho é apresentar um perfil das pacientes com endometriose, estadio e resultados do tratamento entre as pacientes atendidas no nosso Serviço.

Métodos: foram revistos 155 prontuários médicos de pacientes portadoras de endometriose atendidas no periodo de 1991 a 1996 segundo um protocolo preestabelecido.

Resultados: a média de idade das pacientes foi de 31 anos, sendo que a maioria era branca e com ciclos menstruais regulares. Os sintomas mais observados foram a dismenorréia, dor pélvica e infertilidade, sendo esses sinais e sintomas de grau leve na maioria dos casos. Os métodos diagnósticos utilizados foram a laparoscopia ou ultra-sonografia. Houve concordância entre a ultra-sonografia e a laparoscopia em $96 \%$ das vezes. Em $28 \%$ dos casos houve necessidade de realização de laparotomia para elucidação diagnóstica e/ou tratamento. O achado de endometrioma ocorreu em 37\% das vezes e em apenas $74 \%$ das biópsias das lesões houve a confirmação de endometriose pelo estudo anatomopatológico. Houve melhora significativa com o tratamento clinico independente da droga utilizada, com melhora dos sintomas em cerca de $50 \%$ delas. Foram realizadas técnicas de fertilização assistida em 34 pacientes, das quais $80 \%$ foram fertilização in vitro (FIV), com taxa de $27 \%$ de gravidez/paciente.

Conclusões: o método diagnóstico de escolha deve ser a laparoscopia, embora a ultrasonografia apresente um alto indice de concordância. O estudo anatomopatológico das lesões deve ser utilizado como método auxiliar no diagnóstico, pois ele não é positivo em todos os casos. O tratamento clinico com reprodução assistida é uma boa opção terapêutica, principalmente com o uso de FIV.
\end{abstract}

PALAVRAS-CHAVE: Endometriose. Fertilização in vitro. Agonista do GnRH. Laparoscopia.

Departamento de Ginecologia e Obstetrícia do Hospital das Clínicas da Faculdade de Medicina de Ribeirão Preto da Universidade de São Paulo

Correspondência:

Marcos Dias de Moura

Departamento de Ginecologia e Obstetrícia

Faculdade de Medicina de Ribeirão Preto

14048-900 - Ribeirão Preto - SP

Tel: (016) 633-9633

E-mail: <mddmoura@fmrp.usp.br>

\section{Introdução}

A endometriose é uma patologia caracterizada pela presença de tecido endometrial de caráter benigno fora da cavidade uterina. Sua incidência em mulheres inférteis pode chegar até $25 \%$, aumentando para $70 \%$ em mulheres com dor pélvica crônica. Em mulheres assintomáticas a 
incidência é de 1 a $2 \%{ }^{4,5}$.

Há relatos de endometriose associada a anomalias congênitas obstrutivas (estenose ou atresia cervical, agenesia de vagina ou himen imperfurado) em adolescentes. É pouco freqüente antes da menarca e após a menopausa. Gravidez precoce e gestações sucessivas parecem prevenir o desenvolvimento da doença. Raramente é observada em mulheres amenorréicas. Pode estar associada a patologias estrogênio-dependentes como miomatose uterina e hiperplasia endometrial ${ }^{4}$.

Com a endometriose, ocorrem disfunção ovulatória com associação de hiperprolactinemia, sindrome da luteinização do folículo não-roto (LUF) e alterações da fase lútea. Há produção contínua de progesterona até a fase folicular seguinte. Diversos estudos relatam interferência com o desenvolvimento da gestação, com alteração da clivagem e do desenvolvimento embrionário, com conseqüente elevação do risco de abortamentos espontâneos ${ }^{9}$.

O diagnóstico baseia-se na anamnese, no exame físico e nos exames complementares. Dentre os exames complementares, podem ser realizados a ultra-sonografia com transdutores vaginais, dopplervelocimetria colorida, ultrasonografia transvaginal tridimensional, tomografia computadorizada, ressonância nuclear magnética, laparoscopia e dosagens de marcadores como o CA-125, proteína $\mathrm{C}$ reativa e anticorpos anticardiolipina ${ }^{1}$.

O tratamento clínico hormonal visa inibir a produção de gonadotrofinas, anulando a esteroidogênese e, subseqüentemente, liquefazer, necrosar e absorver os implantes. O tratamento pode ser realizado com a administração contínua de anticoncepcionais orais (ACO), progestagênio sintético ou análogos de GnRH. Além disso, para resolução da infertilidade associada, as pacientes podem ser submetidas a técnicas de reprodução assistida como a inseminação intra-uterina (IUI) ou fertilização in vitro (FIV), esta última com resultados mais promissores.

Assim sendo, o objetivo do presente trabalho foi o de realizar uma avaliação retrospectiva dessa patologia e seu manejamento em pacientes atendidas no Hospital das Clínicas da Faculdade de Medicina de Ribeirão Preto, sendo avaliados dados relativos aos aspectos clínicos de sintomatologia e terapêutica.

\section{Pacientes e Métodos}

Foram avaliados 155 prontuários médicos de pacientes portadoras de endometriose atendidas no HCFMRP - USP no período de 1991 a 1996. Com os dados recolhidos do levantamento dos prontuários médicos foi possivel a verificação dos seguintes aspectos:

1- idade com que as pacientes do nosso serviço começam a se queixar de sintomas e quais são eles;

2- raça;

3- idade em que ocorreu a menarca;

4- ciclos menstruais e a compatibilidade com o esperado para estas pacientes;

5- infertilidade associada ao processo;

6- outras patologias associadas ou cirurgia abdominal anterior;

7- métodos mais utilizados para diagnosticar endometriose;

8- percentual de pacientes que apresentaram endometrioma;

9- classificação prevalente da endometriose;

10- tratamento mais utilizado pelo nosso serviço e os resultados obtidos;

11- ocorrência de gravidez posteriormente ao tratamento

As pacientes foram comparadas quanto aos diferentes regimes de terapia, quais sejam o uso de drogas progestagênicas (gestrinona e danazol) e uso de agonistas de GnRH (leuprolida, triptorelin ou goserelin), bem como o resultado da terapêutica cirúrgica e o da terapêutica clínica associada.

\section{Resultados}

As pacientes que procuraram o nosso serviço tinham em média 31 anos, com faixa de variação de 18 a 50 anos. A média de idade da menarca foi de 12 anos, variando de 9 a 19 anos. Houve prevalência da cor branca sobre a cor negra.

Cerca de $74 \%$ das pacientes relatavam ciclos menstruais regulares (considerado regular o intervalo de 25 a 32 dias de duração), ao passo que apenas $31 \%$ apresentaram ciclos menstruais irregulares. A duração da menstruação apresentou ampla variação, de 1 a 10 dias.

Em relação à sintomatologia, prevaleceu a queixa de dismenorréia. A dor pélvica foi relatada 
por $48 \%$ das pacientes, dado semelhante à queixa de dispareunia (43\%). A sinusorragia esteve presente em apenas $7 \%$ dos casos. Foi observado alto percentual de infertilidade nessas pacientes (58\%), das quais $76 \%$ tinham infertilidade primária e $24 \%$ secundária. Foram descritos casos de cirurgias prévias em $37 \%$ das pacientes e a ocorrência de nódulo endometriótico em cicatriz cirúrgica em cerca de $4 \%$ dos casos (Tabela 1).

Tabela 1 - Sinais e sintomas apresentados por pacientes com endometriose

\begin{tabular}{lcc}
\hline Sinais e Sintomas & n/Total & Percentual \\
\hline Dismenorréia & $127 / 149$ & $85 \%$ \\
Dor pélvica & $70 / 146$ & $48 \%$ \\
Dispareunia & $64 / 151$ & $43 \%$ \\
Sinusorragia & $11 / 151$ & $7 \%$ \\
Esterilidade & $88 / 153$ & $58 \%$ \\
- Primária & $68 / 88$ & $76 \%$ \\
- Secundária & $20 / 88$ & $24 \%$ \\
Cirurgia Prévia & $58 / 155$ & $37 \%$ \\
Nódulo Abdominal & $6 / 155$ & $4 \%$ \\
\hline
\end{tabular}

Foram encontradas algumas doenças associadas em $42 \%$ dos casos, mas a grande maioria das pacientes não apresentavam nenhuma patologia ginecológica associada à endometriose. Apenas a miomatose uterina teve presença significativa, sendo os demais achados provavelmente ocasionais e sem relevância clínica.

Dentre os métodos diagnósticos utilizados (ultra-sonografia, laparoscopia e laparotomia), demonstrou-se que em $92 \%$ dos casos foi utilizada a ultra-sonografia (US) e em $71 \%$ empregou-se a laparoscopia diagnóstica. Em $65 \%$ dos casos foi usada a associação de ultra-sonografia e laparoscopia e em $28 \%$ a laparotomia. Apenas 8 pacientes $(5,2 \%)$ foram submetidas aos 3 métodos diagnósticos.

Das 155 pacientes com endometriose, 37 pacientes apresentavam resultado de exame ultrasonográfico ignorado ou não foram submetidas a esse exame. Assim sendo, 118 pacientes apresentavam laudo ultra-sonográfico compativel com endometriose ou outro resultado (considerado "normal"). Quando se comparou o laudo ultrasonográfico com o resultado da laparoscopia e/ou laparotomia observou-se que houve concordância apenas em $41 \%$ delas (48/118), como se pode verificar na Tabela 2. Dentre as pacientes com diagnóstico de endometriose, a concordância da inspeção visual com o laudo anatomopatológico ocorreu em $74 \%$ de 58 pacientes submetidas a biópsia das lesões sugestivas de endometriose à laparoscopia e/ou laparotomia, como mostra a Tabela 3.

Tabela 2 - Comparação entre o diagnóstico ultra-sonográfico e o obtido pela laparoscopia e laparotomia.

\begin{tabular}{lccc} 
Ultra-sonografia & Laparotomia e/ou & Laparoscopia & Total \\
\cline { 2 - 3 } & Normal & Endometriose & \\
\hline Endometriose & 2 & 45 & 47 \\
Normal* & 3 & 68 & 71 \\
\hline
\end{tabular}

* Normal = Não sugestivo de endometriose

Tabela 3 - Resultado dos exames histológicos das biópsias sugestivas de endometriose à laparoscopia e/ou laparotomia.

\begin{tabular}{lrc} 
Anatomopatológico & n & Percentual \\
\hline Normal & 2 & $3 \%$ \\
Endometriose & 43 & $74 \%$ \\
Adenomiose/Miomatose & 6 & $10 \%$ \\
Outros (cistos, pólipos, coágulo, fibrose) & 7 & $13 \%$ \\
Total & 58 & $100 \%$
\end{tabular}

O estágio da endometriose foi baseado na classificação revisada da Sociedade Americana de Medicina Reprodutiva (ASRM) ${ }^{8}$. Dentre as 149 pacientes analisadas segundo as normas da ASRM, $58 \%$ dos casos foram classificados como endometriose de grau leve, $25 \%$ de grau moderado e $15 \%$ de grau grave. Apresentaram endometriose adicional $7 \%$ das pacientes, não-classificada como grau leve, moderado ou grave. Entende-se por endometriose adicional a presença de lesões em intestino, trato urinário, trompas, vagina, cérvice uterino, pele, etc. Quatro pacientes apresentaram apenas endometriose adicional, sendo de localização vaginal, com nódulo abdominal, vesical e na fossa ilíaca esquerda, respectivamente, como pode-se verificar na Tabela 4.

Tabela 4 - Classificação da endometriose segundo critérios da ASRM.

\begin{tabular}{lrr}
\hline Classificação & n & Percentual \\
\hline Leve ou mínima & 86 & $58 \%$ \\
Moderada & 37 & $25 \%$ \\
Grave & 22 & $15 \%$ \\
Adicional & 4 & $2 \%$ \\
Total & 149 & $100 \%$ \\
\hline
\end{tabular}


O tratamento utilizado foi clínico (com a administração contínua de $\mathrm{ACO}$, progestagênio sintético ou análogos de GnRH) ou cirúrgico, que incluiu a cauterização dos focos de endometriose, lise de aderências, ressecção do endometrioma, salpingoplastia e/ou outros como histerectomia, salpingectomia e ooforectomia. Das 155 pacientes, cerca de $17 \%$ foram submetidas apenas ao tratamento clínico, $28 \%$ ao tratamento cirúrgico e $55 \%$ ao tratamento combinado clínico e cirúrgico.

O tratamento clínico foi analisado quanto à evolução das queixas da paciente e ocorrência de gestações espontâneas posteriores. Também comparamos a eficácia dos diferentes medicamentos utilizados (Tabela 5). Das 101 pacientes que foram submetidas ao tratamento clínico, houve melhora ou estabilização do quadro em aproximadamente $50 \%$ dos casos, sendo que nenhuma das formas de tratamento clínico mostrou-se superior a outro no tocante aos sintomas da endometriose. Também não houve relação entre a melhora e o grau de endometriose que a paciente apresentava, nem entre o grau de endometriose e a presença ou não de patologias associadas que pudessem interferir na resposta clinica.

Tabela 5 - Resultado do tratamento clínico da endometriose. São apresentados o número de pacientes e a porcentagem.

\begin{tabular}{lllll}
\hline $\begin{array}{l}\text { Tratamento } \\
\text { clínico }\end{array}$ & $\begin{array}{l}\text { Melhora } \\
\text { clínica }\end{array}$ & $\begin{array}{l}\text { Estável (sem } \\
\text { efeito) }\end{array}$ & $\begin{array}{l}\text { Gravidez } \\
\text { espontânea }\end{array}$ & Total \\
\hline GnRHa* & $18(53 \%)$ & $12(35 \%)$ & $4(12 \%)$ & $34(22 \%)$ \\
Gestrinona & $52(58 \%)$ & $30(33 \%)$ & $8(9 \%)$ & $90(58 \%)$ \\
$\begin{array}{l}\text { GnRHa } \\
+ \text { Gestrinona }\end{array}$ & $8(42 \%)$ & $10(53 \%)$ & $1(5 \%)$ & $19(12 \%)$ \\
$\begin{array}{l}\text { Danazol } \\
\begin{array}{l}\text { GnRHa } \\
+ \text { Gestrinona }\end{array}\end{array}$ & $4(44 \%)$ & $5(56 \%)$ & 0 & $9(6 \%)$ \\
+ Danazol & $1(33 \%)$ & $2(67 \%)$ & 0 & $3(2 \%)$ \\
Outros** & $16(46 \%)$ & $18(52 \%)$ & $1(3 \%)$ & $35(23 \%)$ \\
\hline
\end{tabular}

* GnRHa: Análogos do GnRH (triptorelin, leuprolida ou goserelin)

** Outros: Acetato de medroxiprogesterona (Depoprovera ou Farlutal); utilizados isoladamente ou em associação com os demais.

Das 155 pacientes analisadas, 40 (26\%) foram submetidas à técnica de fertilização assistida. Destas, 14 pacientes (33\%) tentaram IUI, ao passo que 32 pacientes $(77 \%)$ foram submetidas à FIV (6 pacientes realizaram IUI e FIV). Analisando as pacientes que realizaram apenas um tipo de tratamento, encontramos 7 gestações no grupo da FIV e 3 no grupo da IUI, conforme observado na Tabela 6.
Tabela 6 - Resultados do tratamento com técnicas de reprodução assistida em 34 pacientes portadoras de endometriose.

\begin{tabular}{lcr}
\hline Tipo & Pacientes & Gravidez* \\
\hline Inseminação intra-útero & 8 & $3(37 \%)$ \\
Fertilização in vitro & 26 & $7(27 \%)$ \\
Total & 34 & $10(29 \%)$ \\
\hline
\end{tabular}

* ( ) = Taxa de gravidez/paciente

\section{Discussão}

Os nossos dados assemelham-se aos encontrados na literatura, na qual se observa maior prevalência entre mulheres na quarta década de vida, sendo menos freqüente na raça negra. $\mathrm{O}$ quadro clínico é variável, sendo a dor pélvica, a dispareunia e a infertilidade a triade típica mais freqüentemente encontrada. A dor pélvica é cíclica e progressiva, podendo piorar nos períodos prémenstruais, persistir após o término do fluxo menstrual e ser acompanhada de dor lombar ${ }^{5}$. O principal mecanismo causador dos sintomas apresentados pelas pacientes parece ser a liberação de prostaglandinas e interleucinas com aumento da pressão intra-uterina. Menorragia e "spottings" pré-menstruais também são descritos na literatura, embora com menor prevalência. No presente trabalho, foi possivel observar que a sinusorragia ocorreu em apenas $7 \%$ dos casos, sendo a dor pélvica, a dispareunia e a infertilidade os achados mais freqüentes.

A infertilidade, diagnosticada em $58 \%$ das nossas pacientes, pode ser devida a fatores mecânicos relacionados à motilidade tubária inadequada em função de fibrose e processos aderenciais, além de comprometimento ovariano ${ }^{5}$. Focos minimos de endometriose podem alterar a função tubária em virtude do aumento das prostaglandinas no fluido peritoneal, mesmo que a endometriose seja minima ou leve. As prostaglandinas alteram a motilidade tubária, os mecanismos de ruptura folicular, a função do corpo lúteo e a implantação. Diversos estudos relatam também interferência com o desenvolvimento da gestação, com alteração da clivagem e do desenvolvimento embrionário, com conseqüente elevação do risco de abortamentos espontâneos ${ }^{9}$.

Dentre os métodos diagnósticos, a laparoscopia é o mais indicado para obtenção do diagnóstico de certeza. As lesões são mais comumente encontradas nos ovários ou peritôneo pélvico, contêm material sanguinolento e são de 
cor violácea, marrom ou preta. Podem ser encontradas lesões vermelhas vivas e brancas nacaradas, além de aderências a órgãos adjacentes de caráter mais denso se comparadas às causadas por processos inflamatórios. Microscopicamente, a endometriose é revelada por glândulas endometriais típicas e estroma, eritrócitos, deposição de hemossiderina e macrófagos, além de tecido conjuntivo fibroso contendo células inflamatórias. Focos inativos podem apresentar hemossiderina, calcificações e vasos. Todos essas lesões foram encontradas durante a laparoscopia ou a laparotomia diagnóstica. A biópsia também tem seu valor limitado, principalmente nos casos de lesões mais antigas, com cicatriz e retrações peritoneais. Na nossa casuística, apenas $74 \%$ dos casos apresentavam biópsia compatível com endometriose. Este dado é semelhante ao de Bergqvist ${ }^{3}$, que salienta que a correlação anatomopatológica é observada geralmente nas lesões mais ativas. Assim sendo, o estudo anatomopatológico das lesões deve ser utilizado como método auxiliar no diagnóstico pois ele não é positivo em todos os casos.

Outro método diagnóstico é a dosagem do CA125, que é uma glicoproteína produzida no epitélio celômico durante o desenvolvimento embrionário. A elevação deste marcador em mulheres com endometriose é decorrente de sua presença em maior concentração no endométrio ectópico quando comparado ao tópico e à reação inflamatória associada ao processo, ocasionando alteração da capilaridade do endotélio e facilitando a chegada deste marcador à circulação ${ }^{2,7}$. Diversos estudos ${ }^{2,6}$ mostraram aumento mais significativo em casos de doença avançada em comparação com a inicial e por isso se indicaria a laparoscopia somente com a elevação do CA-125 sérico acompanhada de dor pélvica. Quando usado para avaliação de resposta terapêutica, valores inferiores a $16 \mathrm{U} / \mathrm{ml}$ ou quedas superiores a $40 \%$ dos valores anteriormente mensurados confirmariam a boa resposta.

Os tratamentos clínicos hormonais realizados foram a administração de análogos do $\mathrm{GnRH}$, de ACO continuamente ou progestogênio sintético. Os resultados foram satisfatórios sob o ponto de vista de melhora do quadro de que a paciente vinha se queixando, mas não houve desaparecimento das queixas na maioria dos casos analisados. Os resultados quanto a melhora da infertilidade com o uso dos análogos do GnRH exclusivamente ou previamente a técnicas de reprodução assistida são controversos, sendo que a taxa de concepção varia de 30 a $52 \%$ após o tratamento com os análogos ${ }^{5}$.

A FIV é um método de grande valia para o tratamento da infertilidade. A endometriose parece ter um efeito negativo nos resultados de FIV e as fases mais avançadas estão correlacionadas com menores taxas de sucesso ${ }^{4}$. Alguns autores preconizam o uso prévio de análogos do $\mathrm{GnRH}$ com a finalidade de aumentar o número de oócitos, a taxa de fertilização e a taxa de gravidez, embora outros estudos não confirmem esses resultados ${ }^{10,11}$. Nossos dados de $37 \%$ de gravidez com IUI e $27 \%$ com a FIV são semelhantes aos obtidos na população sem endometriose, mas superiores aos relatados quando se emprega o tratamento clínico exclusivo.

\section{Agradecimentos}

Agradecemos a Dra. Adriana Campolungo, Dra. Sonia Martins Unzer, Sra. Maria Cristina Picinato Medeiros de Araújo, Sra. Marilda Hatsumi Yamada Dantas, Sra. Maria Aparecida Carneiro Vasconcelos, Sra. Sandra Aparecida Cavichiollo Vianna e Sra. Albina Verceze Bortolieiro, cujas colaborações foram importantes para a realização desse trabalho.

Este Trabalho foi realizado com apoio da CNPq e do Projeto PIBIC para TNP.

\section{SUMMARY}

Purpose: to present a profile of endometriosis, stages and results of treatment among the patients seen in our Service. Methods: the medical records of 155 patients with endometriosis treated during the period from 1991 to 1996 according to a preestablished protocol were examined.

Results: mean patient age was 31 years, most patients were white, with regular menstrual cycles. The most frequently observed symptoms were dysmenorrhea, pelvic pain and infertility and were mild in most of the cases. The diagnostic methods utilized were laparoscopy or echography. There was agreement between echography and laparoscopy in 96\% of cases. Laparotomy was required in $28 \%$ of cases for diagnostic elucidation and/or treatment. Endometrioma was detected in $37 \%$ of the cases and endometriosis was confirmed in only $74 \%$ of the biopsies from the lesion by anatomopathological examination. There was a significant improvement with clinical treatment regardless of the drug used, with improved symptoms in approximately $50 \%$ of the patients. Assisted fertilization was performed in 34 patients, consisting of in vitrofertilization (IVF) for $80 \%$ of them, with a $27 \%$ pregnancy/transfer rate.

Conclusions: the diagnostic method of choice should be laparoscopy, although echography presented a high rate of agreement. Anatomopathological examination of the lesions should be used as an adjuvant method in the diagnosis since it is not confirmatory in all of the cases. Clinical treatment with assisted reproductive technologies is a good therapeutic option, especially with the use of IVF.

KEY WORDS: Endometriosis. In vitro fertilization. GNRH agonist. Laparoscopy. 


\section{Referências}

1. Abrão MS, Oliveira R, Soares JB, Porto RCP, Fonseca AM, Tognotti E, et al. Humoral immunological alterations in endometriosis. World Congress on Endometriosis; 1992, Brussels, 32-O.

2. Barbieri RL. CA-125 in patients with endometriosis. Fertil Steril 1986; 45:767-9.

3. Bergqvist A. The relationship between endometriotic lesions and the disease endometriosis. Hum Reprod 1995; 10: 11-2.

4. Brosens IA. New principles in the management of endometriosis. Acta Obstet Gynecol Scand (Suppl) 1994; 159:18-21.

5. Brosens IA. Endometriosis. Current issues in diagnosis and medical management. J Reprod Med 1998;43 (3 suppl):281-6
6. Fedelle L, Arcaini L, Vercellini P, Bianchi S, Candianni GB. Serum CA125 measurements in the diagnosis of endometriosis recurrence. Obstet Gynecol 1988; 72: 19-22.

7. Pittaway DE, Fayez JA. Serum CA-125 antigen levels increase during menses. Am J Obstet Gynecol 1987; 156: 75-6.

8. Revised American Society for Reproductive Medicine classification of endometriosis: 1996. Fertil Steril 1997; 67:817-21.

9. Thomas E. Endometriosis: modern approaches. Practitioner 1991;235:818-22.

10.Thomas EJ, Cooke ID. Successful treatment of asymptomatic endometriosis: does it benefit infertile women? Br Med J 1987; 294:1117-9.

11.Tummon IS, Colwell KA, Mackinnon CJ, Nisker JA, Yuzpe AA. Abbreviated endometriosis-associated infertility correlates with in vitro fertilization success. J In Vitro Fertiliz Embryo Transf 1991; 8:149-53.

\section{COMUNICADO AOS ASSOCIADOS E LETTORES}

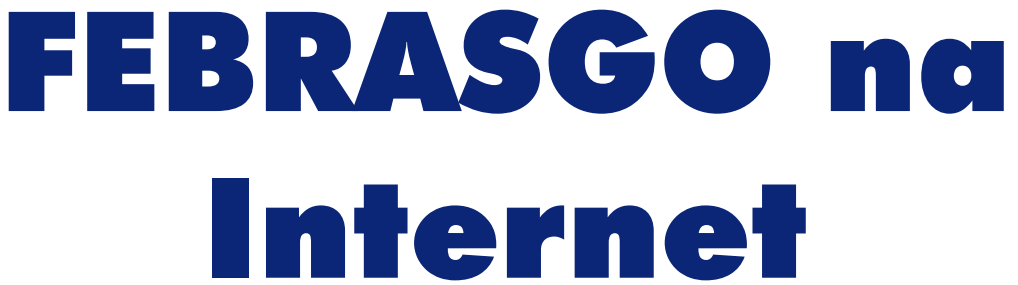

Para informações e consultas temos à disposição quatro endereços eletrônicos:

\section{febrasgopresidencia@alphanet.com.br secretaria_executiva@febrasgo.org.br publicacoes@febrasgo.org.br tego_habilitacao@febrasgo.org.br}

\section{Home page: www.febrasgo.org.br}

